\title{
Erratum
}

\section{A Novel Approach for Pathway Analysis of GWAS Data Highlights Role of BMP Signaling and Muscle Cell Differentiation in Colorectal Cancer Susceptibility - Erratum}

\author{
Aniket Mishra and Stuart MacGregor \\ Genetics and Epidemiology of Colorectal Cancer Consortium (GECCO) and the Colorectal Cancer Family Registry (CCFR), \\ and Statistical Genetics, QIMR Berghofer Medical Research Institute, Brisbane, Queensland, Australia
}

doi: 10.1017/thg.2016.100. Published by Cambridge University Press, 20 January 2017

The publishers regret to announce that the affiliation for the above paper was incorrectly inserted. The correct affiliation is below:

Aniket Mishra ${ }^{1}$, Genetics and Epidemiology of Colorectal Cancer Consortium (GECCO) and the Colorectal Cancer Family Registry (CCFR), and Stuart MacGregor ${ }^{1}$

${ }^{1}$ Statistical Genetics, QIMR Berghofer Medical Research Institute, Brisbane, Queensland, Australia

\section{Reference}

Mishra, A., Genetics, \& Epidemiology of Colorectal Cancer Consortium (GECCO), the Colorectal Cancer Family Registry (CCFR), \& MacGregor, S. (2017) A novel approach for pathway analysis of GWAS data highlights role of BMP signaling and muscle cell differentiation in colorectal cancer susceptibility. Journal of Twin Research and Human Genetics, 20, 1-9. 